\title{
Reorganization of Anatomical Connectome following Electroconvulsive Therapy in Major Depressive Disorder
}

\author{
Jinkun Zeng, ${ }^{1}$ Qinghua Luo, ${ }^{1}$ Lian Du, ${ }^{1}$ Wei Liao, ${ }^{2}$ Yongmei Li, ${ }^{3}$ Haixia Liu, ${ }^{4}$ Dan Liu, \\ Yixiao Fu, ${ }^{1}$ Haitang Qiu, ${ }^{1}$ Xirong Li, ${ }^{1}$ Tian Qiu, ${ }^{1}$ and Huaqing Meng ${ }^{1}$ \\ ${ }^{1}$ Department of Psychiatry, The First Affiliated Hospital of Chongqing Medical University, Chongqing 400016, China \\ ${ }^{2}$ Center for Cognition and Brain Disorders and the Affiliated Hospital, Hangzhou Normal University, Hangzhou 310015, China \\ ${ }^{3}$ Department of Radiology, The First Affiliated Hospital of Chongqing Medical University, Chongqing 400016, China \\ ${ }^{4}$ Medical Psychology Department, The Third Military Medical University, Chongqing 400016, China \\ Correspondence should be addressed to Huaqing Meng; mhq99666@126.com
}

Received 29 April 2015; Revised 1 July 2015; Accepted 13 July 2015

Academic Editor: Martin Walter

Copyright (C) 2015 Jinkun Zeng et al. This is an open access article distributed under the Creative Commons Attribution License, which permits unrestricted use, distribution, and reproduction in any medium, provided the original work is properly cited.

\begin{abstract}
Objective. Electroconvulsive therapy (ECT) is considered one of the most effective and fast-acting treatment options for depressive episodes. Little is known, however, about ECT's enabling brain (neuro)plasticity effects, particular for plasticity of white matter pathway. Materials and Methods. We collected longitudinal diffusion tensor imaging in the first-episode, drug-naïve major depressive disorder (MDD) patients $(n=24)$ before and after a predefined time window ECT treatment. We constructed large-scale anatomical networks derived from white matter fiber tractography and evaluated the topological reorganization using graph theoretical analysis. We also assessed the relationship between topological reorganization with improvements in depressive symptoms. Results. Our investigation revealed three main findings: (1) the small-worldness was persistent after ECT series; (2) anatomical connections changes were found in limbic structure, temporal and frontal lobes, in which the connection changes between amygdala and parahippocampus correlate with depressive symptom reduction; (3) significant nodal strength changes were found in right paralimbic network. Conclusions. ECT elicits neuroplastic processes associated with improvements in depressive symptoms that act to specific local ventral frontolimbic circuits, but not small-world property. Overall, ECT induced topological reorganization in large-scale brain structural network, opening up new avenues to better understand the mode of ECT action in MDD.
\end{abstract}

\section{Introduction}

Patients with major depressive disorder (MDD) typically experience persistent depressed/sad mood and are highly debilitating [1]. Although antidepressant medications and psychotherapeutic treatments are currently available in many patients, electroconvulsive therapy (ECT) is thought to be the most effective and fast-acting remission for depressive episodes $[2,3]$. However, little is known about ECT's enabling brain (neuro)plasticity effects [4], particular for plasticity of white matter pathway [5].

Accumulated evidence suggests that the act of ECT on brain structure associated with clinical state and treatment response in MDD [4]. The hippocampal, amygdalar, and striatal subcortical centers have shown increases in gray matter volume after ECT series, suggesting ECT-induced brain structure neuroplasticity related to improved clinical response [6-9]. Furthermore, diffusion tensor imaging (DTI) can quantify the fiber orientation and integrity of white matter (WM) pathway within neural network. Early specific regions of interest- (ROI-) based DTI studies suggest a trend of increased WM microstructure (fractional anisotropy [FA]) in the hippocampal formation and frontal and temporal lobes following ECT treatment [10, 11]. Recently, whole-brain DTI work found increases of FA in dorsal frontolimbic circuits that are modulated by ECT therapy and relate to therapeutic response [12]. Taken together, MDD do not result from a deficit in a single brain region and local neuronal circuits which contributed to ECT-related structural plasticity. However, it is likely that a large-scale network perspective 
is necessary to explain their complex etiology and ECT's enabling brain neuroplasticity.

The human brain is structurally and functionally organized into connectome $[13,14]$. Depression is also associated with abnormal topological organization of brain networks [15-17], which are valuable for diagnosis neuromarkers and treatment evaluation. In the current study, we collected longitudinal DTI in the first-episode, drug-naïve MDD patients before and after a predefined time window ECT treatment. We constructed large-scale anatomical networks derived from WM fiber tractography and evaluated the dynamic alteration of global, nodal topological characteristics and the strength of each connection using graph theoretical analysis. We aimed to directly answer whether neuroplasticity of ECT related to large-scale anatomical connectome. We also assessed the relationship between topological organization with depressive severity during the ECT series, to test above ECT-related network plasticity would accounts for improvements in depressive symptoms.

\section{Materials and Methods}

2.1. Participants. All examinations were carried out under the guidance of the Declaration of Helsinki 1975. The protocol was reviewed and approved by the Local Medical Ethics Committee of the First Affiliated Hospital of Chongqing Medical University. All these patients were first-episode MDD, without any treatment history before. The diagnosis of MDD was confirmed with a structured clinical interview for DSM-IV-TR Disorders (SCID-I/P, Chinese version) [18], along with scores $\geq 21$ on the 24-item Hamilton Rating Scale for Depression (HAMD) [19]. According to the clinical guidelines of the Canadian Network for Mood and Anxiety Treatments, ECT could be considered as first-line treatment for depression with acute suicidal ideation, psychotic features, and catatonia [20]. Consequently, all the patients were required to have at least one of the above three characteristics. Patients were excluded if they (i) had any history of alcohol or drug abuse, neurological or serious physical disease, and morphological anomaly of the brain and (ii) had any surgical electronic or metal implants. MDD patients were scanned before one day of starting the ECT sessions. All the patients were scanned for the second time at least $>1$ day after eight ECT sessions. We did not control the medicine of subjects during this study, which was decided by their clinician independently. After the first ECT, most patients were given antidepressants over the course of ECT.

2.2. ECT Procedures. The patients underwent modified bitemporal ECT using a brief-pulse, constant current apparatus Thymatron (TM) DGx (Somatics LLC, Lake Bluff, IL, USA) at the Department of Psychiatry of the First Affiliated Hospital of Chongqing Medical University. The first three ECT administrations occurred on consecutive days, and the remaining ECT administrations were conducted every other day with a break of weekends until at least eight times of ECT [22]. After that, ECT treatments were continued if clinical depressive symptoms had not improved sufficiently, which were also decided by a clinician, but lasted at most 12 times of ECTs. The initial dosage selected was normalized, based on sex, age, and weight. Anesthesia was induced with propofol $(1.5-2 \mathrm{mg} / \mathrm{kg})$ and succinylcholine $(0.5-1 \mathrm{mg} / \mathrm{kg})$. We applied a stimulus dose up to 1.5-2 times above seizure threshold [20].

2.3. Clinical Assessments. The patients received scanning and depressive symptoms assessments at two separate time points: (i) within one day before their first ECT session (preECT) and (ii) the day after completion of eight ECT sessions (post-ECT). Ratings of depressive symptoms were collected at each time point using the 24-item HAMD.

2.4. Image Acquisition and Preprocessing. We acquired imaging data using a 3.0 Tesla MRI system (GE Signa) in the First Affiliated Hospital of Chongqing Medical University. We used foam padding to minimize head motion. We acquired 3D T1weighted anatomical images (repetition time $=8.35 \mathrm{~ms}$, echo time $=3.27 \mathrm{~ms}$, flip angle $=12^{\circ}$, field of view $=240 \times 240 \mathrm{~mm}^{2}$, matrix $=256 \times 256$, slice thickness $=1 \mathrm{~mm}$, and number of slices $=156$ sagittal slices). We also acquired the diffusionweighted images using spin echo-based echo planar imaging sequence ( 25 noncollinear directions, $b=1000 \mathrm{~s} / \mathrm{mm}^{2}$, one volume without diffusion-weighted $b=0 \mathrm{~s} / \mathrm{mm}^{2}$, number of slices $=37$ axial slices, repetition time $=10000 \mathrm{~ms}$, echo time $=86.7 \mathrm{~ms}$, flip angle $=90^{\circ}$, field of view $=256 \times 256 \mathrm{~mm}^{2}$, matrix $=256 \times 256$, slice thickness $=3 \mathrm{~mm}$, and number of averages $=2$ ).

DTI data were preprocessed and analyzed using the Pipeline for Analyzing Brain Diffusion Images toolkit (PANDA; http://www.nitrc.org/projects/panda) [24], which synthesizes procedures in FSL (http://fsl.fmrib.ox.ac.uk/fsl) and the diffusion toolkit. DTI data were coregistered to the B0 image and corrected for distortion induced by eddy currents. Diffusion tensor models were estimated by using the linear least-squares fitting method at each voxel by using the diffusion toolkit. Whole-brain MW fiber tracking was performed in the native diffusion space for each subject by using the fiber assignment with the continuous tracking algorithm. Path tracking proceeded until either the fractional anisotropy was less than 0.15 or the angle between the current and the previous path segment was greater than 35 degrees, as in our previous studies [25-27]. Fibers less than $10 \mathrm{~mm}$ or with obvious false paths were discarded.

2.5. Anatomical Connectome Construction. To determine the nodes of anatomical connectivity networks, we used the automated anatomical labelling (AAL) template [23] to parcellate the whole cerebral cortex into 90 noncerebellar anatomical ROIs. A list of anatomical labels of these ROIs are presented in Table 2. The ROIs were transformed into each subject's native diffusion space. Specifically, we coregistered the individual $3 \mathrm{D}-\mathrm{T} 1$ images to $\mathrm{B} 0$ images and normalized the $3 \mathrm{D} \mathrm{T} 1$ images to the Montreal Neurologic Institute space by a 12-parameter nonlinear transformation. These transformation parameters were inversed and applied to 90 ROIs. 
In this native diffusion space, anatomical connectivity between ROI $i$ and $j$ was defined as the number of fibers connecting $i$ and $j$. We selected fiber number as measurement because it is sensitive to the topological difference between patients with distinct outcomes. The raw network connectivity (fiber number) was scaled to the total volume of ROI $i$ and $j$ [25]. For each subject, the anatomical connectivity matrix " $M$ " had $90 \times 90$ entries, with $A_{i j}$ corresponding to the weighted connectivity between ROI $i$ and $j$, also referred to as the link between nodes $i$ and $j$.

2.6. Graph Theoretic Measures. Graph theory enables the quantification of network topological properties [28]. Graph measures for each individual connectivity matrix were calculated with the Brain Connectivity Toolbox (https://sites.google .com/site/bctnet/). Small-worldness is an optimal architecture balancing the segregation and integration of information, with similar path length $\left(\lambda=L_{\text {net }}^{w} / L_{\text {random }}^{w} \approx 1\right)$ but higher clustering coefficient $\left(\gamma=C_{\text {net }}^{w} / C_{\text {random }}^{w}>1\right)$ than a random network [29]. We evaluated the typical properties of a small-worldness $(\sigma=\gamma / \lambda)$ that is typically larger than one. We selected nodal strength to estimate the topological feature of each node, because its high test-retest reliability [30] and clear neurophysiology relevance [31]. Nodal strength $\left(S_{i}\right)$ was computed as the sum of the weights of all the connections of node $i$. It quantifies the extent to which a node is relevant to the graph.

2.7. Statistical Analysis. To examine how anatomical architectures reorganized following ECT, we performed a paired $t$ test (post-ECT versus pre-ECT) for network measures. The anatomical connectivity edge, small-worldness, and nodal strength were analyzed, respectively. In addition, to explore associations between network measures changes (post-ECT - pre-ECT) and the depressive symptom reduction (pre-ECT - post-ECT HAMD scores) in patients after ECT $(n=22)$, we calculated the Pearson correlation coefficients. We also computed the relationship between the anatomical measures of the pre-ECT data and the duration of depressive episode. As these analyses were exploratory, we used an uncorrected statistical significance level of $P<0.05$.

\section{Results}

3.1. Clinical Data. Twenty-four first-episode, drug-naïve MDD patients (15 female, all right-handed, age [mean \pm SD]: $28.88 \pm 10.77$ years) who received eight ECT series have enrolled (Table 1). Note that two patients' clinical data were missed. Patients' HAMD score before ECT was $28.14 \pm 5.43$, indicating severe depression. After ECT series, depressive symptoms (HAMD score) significantly reduced $\left(T_{21}=11.92\right.$, $P<0.0001$, paired $t$-test) (Table 1 ), indicating excellent therapeutic effects of ECT. Nineteen of the 22 patients $(86.36 \%)$ were ECT responders whose depressive symptoms reduced at least to 50\% compared to pre-ECT HAMD [32]. Ten of the 22 patients (45\%) were in remission - that is, their HAMD scores were $\leq 7-$ after the ECT series. After the first
TABLE 1: Demographic and clinical characteristics of patients.

\begin{tabular}{|c|c|c|c|}
\hline Demographics & & $\operatorname{MDD}(n=24)$ & \\
\hline Age (years) & & $28.88 \pm 10.77$ & \\
\hline Sex (male/female) & & 9/15 & \\
\hline Education (years) & & $11.96 \pm 2.79$ & \\
\hline Age of onset (years) ${ }^{*}$ & & $26.62 \pm 12.12$ & \\
\hline $\begin{array}{l}\text { Suicidal thought or } \\
\text { behavior }(\%)^{*}\end{array}$ & & 77.27 & \\
\hline \multirow[t]{2}{*}{$\begin{array}{l}\text { Duration of depressive } \\
\text { episode (months) }\end{array}$} & & $2.83 \pm 5.43$ & \\
\hline & Pre-ECT & Post-ECT & $P$ value \\
\hline HAMD* & $28.14 \pm 5.43$ & $8.81 \pm 4.20$ & $<0.0001^{\mathrm{a}}$ \\
\hline
\end{tabular}

MDD, major depressive disorder; HAMD, Hamilton Rating Scale for Depression; ECT, electroconvulsive therapy.

The values are illustrated as mean \pm SD.

${ }^{*}$ Two patients' clinical data missed.

${ }^{\text {a }}$ Paired $t$-test.

ECT, 20 of the 22 patients (90.9\%) were given antidepressants over the course of ECT.

3.2. ECT Effects on Topological Reorganization. The anatomical connectome exhibited typical features of small-word topology no matter pre- or post-ECT. However, no significant difference was found between post- and pre-ECT $(T=0.77$, $P=0.447)$.

Eight anatomical connections showed reorganization feature after ECT series $(P<0.01$, corrected). The six increased connections were mainly located in limbic structure, temporal and frontal lobes, for example, amygdala (AMYG) versus parahippocampal gyrus (PHG), inferior temporal gyrus (ITG) versus middle temporal gyrus (MTG), and middle temporal pole (TPOsup) versus orbital inferior frontal gyrus (ORBinf), while the two connections, including anterior cingulate cortex (ACC) versus medial superior frontal gyrus (SFGmed) and precuneus (PCUN) versus superior occipital gyrus (SOG), showed decreased connection. In addition, the connection changes between amygdala and parahippocampus correlated with depressive symptom reduction (Figure 1).

A comparison of post-ECT treatment and pre-ECT treatment on nodal strength revealed significant increases in right PHG $(T=2.207, P=0.037)$ and ORBinf $(T=$ 2.073, $P=0.049$ ) and decreases in left fusiform gyrus (FFG) $(T=-2.285, P=0.031)$ (Figure 2). The nodal strength changes of FFG correlated with depressive symptom reduction (Figure 2).

There is no significant correlation between the anatomical measures of the pre-ECT data and the duration of depressive episode.

\section{Discussion}

We investigated the reorganization mechanism of anatomical connectome, rather than local brain structure alone, in first-episode, drug-naïve MDD patients subsequent to ECT treatments. Our investigation revealed three main findings: 

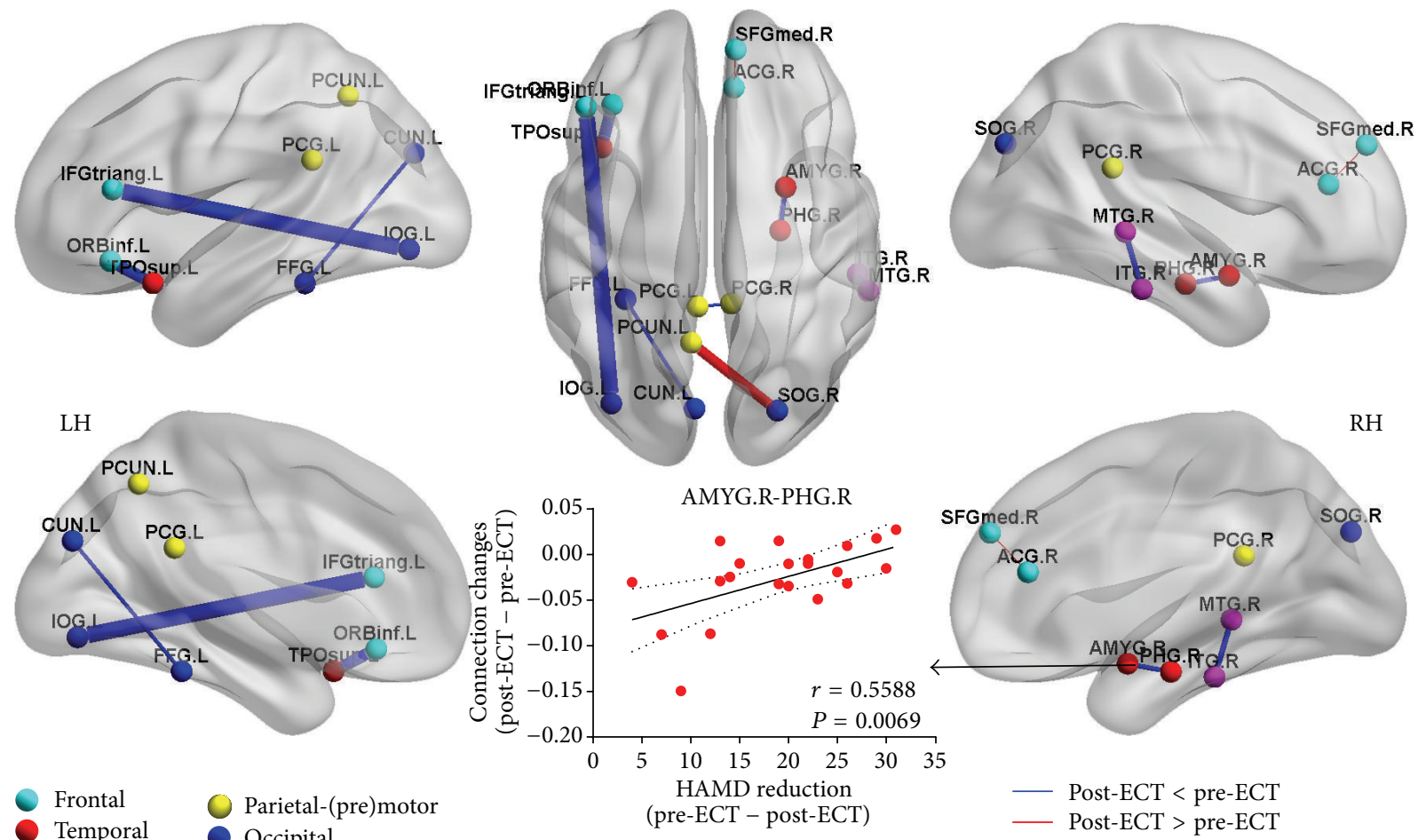

FIGURE 1: Significant differences in anatomical connection between post-ECT and pre-ECT. Nodes (individual ROIs) were differently colored according to the six anatomical modules listed in Table 2. Undirected edges were differently colored according to the significantly larger connection $(P<0.05)$. Nodes and edges are presented on inflated surface maps by BrainNet Viewer (http://www.nitrc.org/projects/bnv) [21]. Scatter-plot indicated the changed connection (post-ECT - pre-ECT) showing significant correlation with the HAMD reduction (pre-ECT - post-ECT).

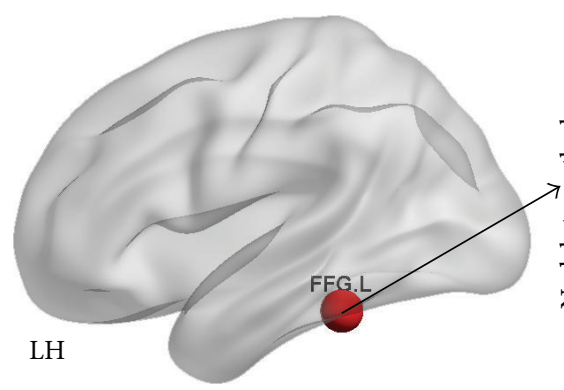

Post-ECT > pre-ECT
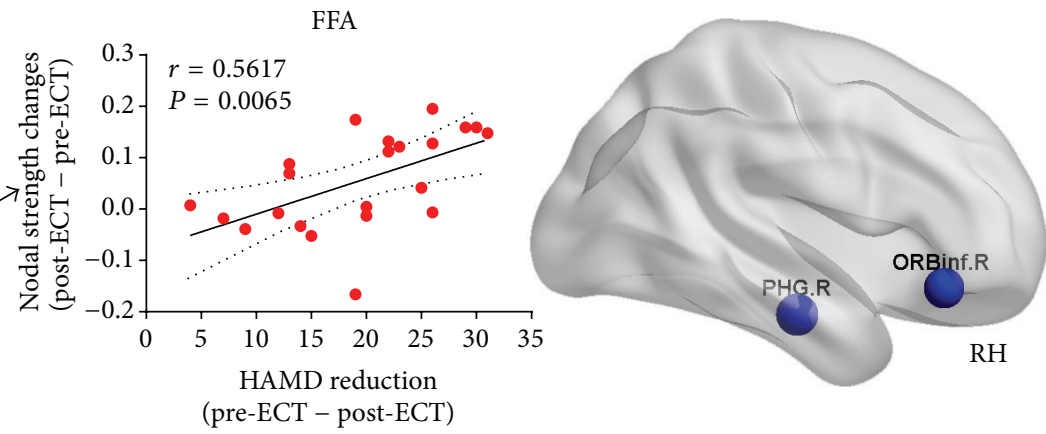

Post-ECT $<$ pre-ECT

FIGURE 2: Significant differences in nodal strength between post-ECT and pre-ECT. Nodes (individual ROIs) were differently colored according to the significantly increased and decreased nodal strength $(P<0.05)$. Nodes are presented on inflated surface maps by BrainNet Viewer (http://www.nitrc.org/projects/bnv) [21]. Scatter-plot indicated the changed nodal strength in fusiform gyrus (post-ECT - pre-ECT) showing significant correlation with the HAMD reduction (pre-ECT - post-ECT).

(1) the small-worldness was persistent after ECT series; (2) significant anatomical connections changes were found in limbic structure, temporal and frontal lobes, in which the connection changes between amygdala and parahippocampus correlated with depressive symptom reduction; and (3) significant nodal strength changes were found in right paralimbic network. These findings would support the that ECT-induced brain structure neuroplasticity relates to improved clinical response [9].

The human brain is a complex network organized with a small-world property (high efficiency at a low wiring cost) [33]. This economic architecture was altered in the patients, suggesting a disturbance of the normal balance of segregation and integration of information in anatomical connectome in 
TABLE 2: Regions of interest (ROI) in the AAL template.

\begin{tabular}{|c|c|}
\hline Region name & Abbreviation \\
\hline \multicolumn{2}{|l|}{ Medial temporal } \\
\hline Amygdala & AMYG \\
\hline Hippocampus & HIP \\
\hline Parahippocampal gyrus & PHG \\
\hline Middle temporal gyrus, temporal pole & TPOsup \\
\hline Superior temporal gyrus, temporal pole & TPOmid \\
\hline \multicolumn{2}{|l|}{ Subcortical } \\
\hline Caudate nucleus & CAU \\
\hline Olfactory cortex & OLF \\
\hline Palladium & PAL \\
\hline Putamen & PUT \\
\hline Thalamus & THA \\
\hline \multicolumn{2}{|l|}{ Occipital } \\
\hline Calcarine fissure & CAL \\
\hline Cuneus & CUN \\
\hline Fusiform gyrus & FFG \\
\hline Lingual gyrus & LING \\
\hline Inferior occipital gyrus & IOG \\
\hline Middle occipital gyrus & MOG \\
\hline Superior occipital gyrus & SOG \\
\hline \multicolumn{2}{|l|}{ Temporal } \\
\hline Heschl gyrus & HES \\
\hline Insula & INS \\
\hline Inferior temporal gyrus & ITG \\
\hline Middle temporal gyrus & MTG \\
\hline Superior temporal gyrus & STG \\
\hline \multicolumn{2}{|l|}{ Frontal } \\
\hline Anterior cingulate cortex & $\mathrm{ACC}$ \\
\hline Inferior frontal gyrus, opercular & IFGoper \\
\hline Inferior frontal gyrus, orbital & ORBinf \\
\hline Inferior frontal gyrus, triangular & IFGtri \\
\hline Superior frontal gyrus, medial orbital & SFGmorb \\
\hline Middle frontal gyrus, orbital & MFGorb \\
\hline Middle frontal gyrus & MFG \\
\hline Superior frontal gyrus, medial & SFGmed \\
\hline Superior frontal gyrus, orbital & SFGorb \\
\hline Superior frontal gyrus & SFG \\
\hline Gyrus rectus & REG \\
\hline \multicolumn{2}{|l|}{ Parietal-(pre) Motor } \\
\hline Rolandic operculum & ROL \\
\hline Angular gyrus & ANG \\
\hline Median cingulate gyrus & MCC \\
\hline Posterior cingulate gyrus & PCC \\
\hline Paracentral lobule & PCL \\
\hline Inferior parietal gyrus & IPG \\
\hline Superior parietal gyrus & SPG \\
\hline Postcentral gyrus & PoCG \\
\hline Precentral gyrus & PreCG \\
\hline Precuneus & PCUN \\
\hline Supplementary motor area & SMA \\
\hline Supramarginal gyrus & SMG \\
\hline
\end{tabular}

The abbreviations used in the study differ slightly from the original abbreviations by Tzourio-Mazoyer et al. [23].

depression $[15,17]$. For the first time, we revealed whether the small-world property is adaptive following the ECT series. Not surprising, the small-worldness was persistent, given that the small-world topology reflects an optimal balance between global integration and local special [34], without evidence for ECT-related change in global topology. Furthermore, the functional connectome is thought to be more flexible, while the anatomical connectome is relatively stable $[25,35]$. We therefore reasoned that the anatomical connectivity network may be less affected by ECT in MDD.

Although the above-mentioned global topology was persistent, the anatomical connections showed reorganization in ventral and dorsal frontolimbic structure following ECT series. Previous cross-sectional MDD studies have consistently shown that increased anatomical connections were mainly located in cortical-limbic network, particularly in the frontolimbic network $[15,36,37]$. These prior findings support a dysregulation between underactive dorsal and overactive ventral frontolimbic circuitry [12]. Though few DTI studies have assessed treatment effects in MDD patients, our longitudinal results was compatible with prior findings. Our results further demonstrate ECT-related changes in the anatomical connections of selected frontolimbic pathways.

The amygdala and hippocampus play a pivotal role in regulation of emotion and in responses to emotion [38, 39]. The effects of ECT on amygdala and hippocampus volume suggest that ECT-induced brain gray matter structure neuroplasticity relates to improved clinical response [69]. We considered the anatomical basis connecting them, rather than alone, because the hippocampus connects with the amygdala and the limbic hypothalamic-pituitary-adrenal (HPA) axis, which are central to the pathophysiology of the $\operatorname{MDD}[9,40]$. In the current work, the fiber number was used to index the anatomical connection, which are closely related to neurite components of synapses (synaptogenesis) in neuroplasticity model [4]. Decreased connection would reflect axonal pruning [41], which regulate the HPA system activity [42]. Furthermore, these connection changes associate with HMDA reduction, suggesting that ECT-related anatomical connection plasticity may contribute to improvements in depressive symptoms.

The post-ECT and pre-ECT comparison of nodal topological characteristic, for example, nodal strength, revealed alterations or neuroplasticity of network hubs in MDD patients. The characteristic of nodal strength is the most fundamental network measure with high reliability [30], neurophysiology relevance [31], and sensitive neuromarkers of MDD [16]. The changes of nodal strength in right hippocampus and inferior frontal lobe, consistent with prior local brain plasticity in ventral frontolimbic structure. Rather unexpectedly, we also observed that the fusiform gyrus presented increases nodal topological characteristics following ECT series. This change was furthermore shown to be positively correlated with depressive symptoms relevance. A more recent meta-analysis in MDD patients pointed to smaller FA in fusiform gyrus that is involved in inferior longitudinal fasciculus [43]. The role of the fusiform gyrus in memory processing and face recognition may contribute to cognitive vulnerability to depression [44]. Our finding of increased nodal strength under this gyrus may provide structural evidence of white matter plasticity in the neural circuit in MDD from a connectome point of view. 
Several methodological limitations need to be addressed. First, in absence of a healthy control group, we would not assess the normalization of aberrant network measures when compared post-ECT depression group and controls [45] and predict the treatment response/remission when compared pre-ECT depression group and controls [46]. Second, the patients were only scanned twice within a predefined time window. More scans would conduce to explore the relation between times of ECT and neuroimaging changes for each patient [47]. In addition, although ECT showed antidepressant therapeutic effect, the ECT-related cognitive side effects have not been considered here, which are worthy of concern in future studies [48]. Finally, although diffusion tensor modal is an effective way to investigate white matter networks, some advanced approaches (e.g., diffusion spectrum imaging) may lead to more accurate tractography results, particularly in areas with cross fibers.

\section{Conclusion}

ECT elicits neuroplastic processes associated with improvements in depressive symptoms that act to specific local ventral frontolimbic circuits, but not global small-world property. ECT-related changes in the anatomical connections of selected amygdala and hippocampus associate with axonal pruning, which regulate the HPA system activity. Our finding of increased nodal strength under fusiform gyrus may provide structural evidence of white matter plasticity in the neural circuit in MDD from a connectome point of view for adapting cognitive vulnerability. Overall, ECT induced topological reorganization in large-scale brain structural network, opening up new avenues to better understand the mode of ECT action in MDD.

\section{Conflict of Interests}

The authors declare that there is no potential conflict of interests regarding the publication of this paper.

\section{Authors' Contribution}

Jinkun Zeng, Qinghua Luo, and Lian Du contributed to data collection and wrote the paper. Lian Du and Wei Liao contributed to data analyses. Wei Liao, Yongmei Li, Haixia Liu, Dan Liu, Yixiao Fu, Haitang Qiu, Xirong Li, and Tian Qiu helped revise the paper. Huaqing Meng designed the experiment and revised the paper. Jinkun Zeng, Qinghua Luo, and Lian Du contributed equally to this work.

\section{Acknowledgments}

This work was supported by the grants from the National Key Clinical Specialist Construction Programs of China ([2013] 544), the Natural Science Foundation of Chongqing Province (no. cstc2014yykfA110030), and the fund of West China Psychiatric Association (wcpafund2013-9).

\section{References}

[1] D. J. Kupfer, E. Frank, and M. L. Phillips, "Major depressive disorder: new clinical, neurobiological, and treatment perspectives," The Lancet, vol. 379, no. 9820, pp. 1045-1055, 2012.

[2] M. Fink, "ECT has proved effective in treating depression," Nature, vol. 403, no. 6772, p. 826, 2000.

[3] H.-P. Spaans, P. Sienaert, F. Bouckaert et al., "Speed of remission in elderly patients with depression: electroconvulsive therapy v. medication," British Journal of Psychiatry, vol. 206, no. 1, pp. 6771, 2015.

[4] F. Bouckaert, P. Sienaert, J. Obbels et al., "ECT: its brain enabling effects: a review of electroconvulsive therapy-induced structural brain plasticity," Journal of ECT, vol. 30, no. 2, pp. 143-151, 2014.

[5] D. P. Devanand, A. J. Dwork, E. R. Hutchinson, T. G. Bolwig, and H. A. Sackeim, "Does ECT alter brain structure?" The American Journal of Psychiatry, vol. 151, no. 7, pp. 957-970, 1994.

[6] P. Nordanskog, U. Dahlstrand, M. R. Larsson, E.-M. Larsson, L. Knutsson, and A. Johanson, "Increase in hippocampal volume after electroconvulsive therapy in patients with depression: a volumetric magnetic resonance imaging study," Journal of ECT, vol. 26, no. 1, pp. 62-67, 2010.

[7] P. Nordanskog, M. R. Larsson, E.-M. Larsson, and A. Johanson, "Hippocampal volume in relation to clinical and cognitive outcome after electroconvulsive therapy in depression," Acta Psychiatrica Scandinavica, vol. 129, no. 4, pp. 303-311, 2014.

[8] I. Tendolkar, M. van Beek, I. van Oostrom et al., "Electroconvulsive therapy increases hippocampal and amygdala volume in therapy refractory depression: a longitudinal pilot study," Psychiatry Research-Neuroimaging, vol. 214, no. 3, pp. 197-203, 2013.

[9] S. H. Joshi, R. T. Espinoza, T. Pirnia et al., "Structural plasticity of the hippocampus and amygdala induced by electroconvulsive therapy in major depression," Biological Psychiatry, 2015.

[10] K. Nobuhara, G. Okugawa, T. Minami et al., "Effects of electroconvulsive therapy on frontal white matter in late-life depression: a diffusion tensor imaging study," Neuropsychobiology, vol. 50, no. 1, pp. 48-53, 2004.

[11] K. Szabo, J. G. Hirsch, M. Krause et al., "Diffusion weighted MRI in the early phase after electroconvulsive therapy," Neurological Research, vol. 29, no. 3, pp. 256-259, 2007.

[12] H. Lyden, R. T. Espinoza, T. Pirnia et al., "Electroconvulsive therapy mediates neuroplasticity of white matter microstructure in major depression," Translational Psychiatry, vol. 4, article e380, 2014.

[13] O. Sporns, G. Tononi, and R. Kötter, "The human connectome: a structural description of the human brain," PLoS Computational Biology, vol. 1, no. 4, article e42, 2005.

[14] A. Fornito, A. Zalesky, and M. Breakspear, "The connectomics of brain disorders," Nature Reviews Neuroscience, vol. 16, no. 3, pp. 159-172, 2015.

[15] M. S. Korgaonkar, A. Fornito, L. M. Williams, and S. M. Grieve, "Abnormal structural networks characterize major depressive disorder: a connectome analysis," Biological Psychiatry, vol. 76, no. 7, pp. 567-574, 2014.

[16] Q. Gong and Y. He, "Depression, neuroimaging and connectomics: a selective overview," Biological Psychiatry, vol. 77, no. 3, pp. 223-235, 2015.

[17] F. Bai, N. Shu, Y. Yuan et al., “Topologically convergent and divergent structural connectivity patterns between patients 
with remitted geriatric depression and amnestic mild cognitive impairment," Journal of Neuroscience, vol. 32, no. 12, pp. 43074318, 2012.

[18] M. B. First, R. L. Spitzer, M. Gibbon, and J. B. W. Williams, Structured Clinical Interview for DSM-IV-TR Axis I DisordersPatient Edition, (SCID-I/P, 11/2002 Revision), New York State Psychiatric Institute, New York, NY, USA, 2002.

[19] C. H. Kellner, R. G. Knapp, G. Petrides et al., "Continuation electroconvulsive therapy vs pharmacotherapy for relapse prevention in major depression: a multisite study from the consortium for research in electroconvulsive therapy (CORE)," Archives of General Psychiatry, vol. 63, no. 12, pp. 1337-1344, 2006.

[20] S. H. Kennedy, R. Milev, P. Giacobbe et al., "Canadian Network for Mood and Anxiety Treatments (CANMAT) Clinical guidelines for the management of major depressive disorder in adults. IV. Neurostimulation therapies," Journal of Affective Disorders, vol. 117, supplement 1, pp. S44-S53, 2009.

[21] M. Xia, J. Wang, and Y. He, "BrainNet Viewer: a network visualization tool for human brain connectomics," PLoS ONE, vol. 8, no. 7, Article ID e68910, 2013.

[22] E. Z. Reininghaus, B. Reininghaus, R. Ille et al., "Clinical effects of electroconvulsive therapy in severe depression and concomitant changes in cerebral glucose metabolism-an exploratory study," Journal of Affective Disorders, vol. 146, no. 2, pp. 290-294, 2013.

[23] N. Tzourio-Mazoyer, B. Landeau, D. Papathanassiou et al., "Automated anatomical labeling of activations in SPM using a macroscopic anatomical parcellation of the MNI MRI singlesubject brain," NeuroImage, vol. 15, no. 1, pp. 273-289, 2002.

[24] Z. Cui, S. Zhong, P. Xu, Y. He, and G. Gong, "PANDA: a pipeline toolbox for analyzing brain diffusion images," Frontiers in Human Neuroscience, vol. 7, article 42, 2013.

[25] Z. Zhang, W. Liao, H. Chen et al., "Altered functional-structural coupling of large-scale brain networks in idiopathic generalized epilepsy," Brain, vol. 134, no. 10, pp. 2912-2928, 2011.

[26] W. Liao, Z. Zhang, Z. Pan et al., "Default mode network abnormalities in mesial temporal lobe epilepsy: a study combining fMRI and DTI," Human Brain Mapping, vol. 32, no. 6, pp. 883895, 2011.

[27] G.-J. Ji, Z. Zhang, Q. Xu, Y.-F. Zang, W. Liao, and G. Lu, "Generalized tonic-clonic seizures: aberrant interhemispheric functional and anatomical connectivity," Radiology, vol. 271, no. 3, pp. 839-847, 2014.

[28] C. J. Stam, "Modern network science of neurological disorders," Nature Reviews Neuroscience, vol. 15, no. 10, pp. 683-695, 2014.

[29] D. J. Watts and S. H. Strogatz, "Collective dynamics of 'smallworld' networks," Nature, vol. 393, no. 6684, pp. 440-442, 1998.

[30] J.-H. Wang, X.-N. Zuo, S. Gohel, M. P. Milham, B. B. Biswal, and Y. He, "Graph theoretical analysis of functional brain networks: test-retest evaluation on short- and long-term resting-state functional MRI data," PLoS ONE, vol. 6, no. 7, Article ID e21976, 2011.

[31] X. Liang, Q. Zou, Y. He, and Y. Yang, "Coupling of functional connectivity and regional cerebral blood flow reveals a physiological basis for network hubs of the human brain," Proceedings of the National Academy of Sciences of the United States of America, vol. 110, no. 5, pp. 1929-1934, 2013.

[32] M. M. Husain, A. J. Rush, M. Fink et al., "Speed of response and remission in major depressive disorder with acute electroconvulsive therapy (ECT): a consortium for research in ECT
(CORE) report," Journal of Clinical Psychiatry, vol. 65, no. 4, pp. 485-491, 2004.

[33] E. Bullmore and O. Sporns, "The economy of brain network organization," Nature Reviews Neuroscience, vol. 13, no. 5, pp. 336-349, 2012.

[34] O. Sporns, G. Tononi, and G. M. Edelman, “Theoretical neuroanatomy: relating anatomical and functional connectivity in graphs and cortical connection matrices," Cerebral Cortex, vol. 10, no. 2, pp. 127-141, 2000.

[35] C.-H. Park, S. Y. Kim, Y.-H. Kim, and K. Kim, "Comparison of the small-world topology between anatomical and functional connectivity in the human brain," Physica A: Statistical Mechanics and Its Applications, vol. 387, no. 23, pp. 5958-5962, 2008.

[36] M. S. Korgaonkar, N. J. Cooper, L. M. Williams, and S. M. Grieve, "Mapping inter-regional connectivity of the entire cortex to characterize major depressive disorder: a whole-brain diffusion tensor imaging tractography study," NeuroReport, vol. 23, no. 9, pp. 566-571, 2012.

[37] P. Fang, L.-L. Zeng, H. Shen et al., "Increased cortical-limbic anatomical network connectivity in major depression revealed by diffusion tensor imaging," PLOS ONE, vol. 7, no. 9, Article ID e45972, 2012.

[38] N. A. Groenewold, E. M. Opmeer, P. de Jonge, A. Aleman, and S. G. Costafreda, "Emotional valence modulates brain functional abnormalities in depression: evidence from a meta-analysis of fMRI studies," Neuroscience and Biobehavioral Reviews, vol. 37, no. 2, pp. 152-163, 2013.

[39] S. J. Russo and E. J. Nestler, “The brain reward circuitry in mood disorders," Nature Reviews Neuroscience, vol. 14, no. 9, pp. 609625,2013

[40] J. S. Snyder, A. Soumier, M. Brewer, J. Pickel, and H. A. Cameron, "Adult hippocampal neurogenesis buffers stress responses and depressive behaviour," Nature, vol. 476, no. 7361, pp. 458-461, 2011.

[41] K. H. Bockhorst, P. A. Narayana, R. Liu et al., "Early postnatal development of rat brain: in vivo diffusion tensor imaging," Journal of Neuroscience Research, vol. 86, no. 7, pp. 1520-1528, 2008.

[42] A. Surget, A. Tanti, E. D. Leonardo et al., "Antidepressants recruit new neurons to improve stress response regulation," Molecular Psychiatry, vol. 16, no. 12, pp. 1177-1188, 2011.

[43] Y. Liao, X. Huang, Q. Wu et al., "Is depression a disconnection syndrome? Meta-analysis of diffusion tensor imaging studies in patients with MDD," Journal of Psychiatry and Neuroscience, vol. 38, no. 1, pp. 49-56, 2013.

[44] G. A. van Wingen, P. van Eijndhoven, H. R. Cremers et al., "Neural state and trait bases of mood-incongruent memory formation and retrieval in first-episode major depression," Journal of Psychiatric Research, vol. 44, no. 8, pp. 527-534, 2010.

[45] C. C. Abbott, T. Jones, N. T. Lemke et al., "Hippocampal structural and functional changes associated with electroconvulsive therapy response," Translational Psychiatry, vol. 4, article e483, 2014.

[46] J. A. van Waarde, H. S. Scholte, L. J. B. van Oudheusden, B. Verwey, D. Denys, and G. A. van Wingen, "A functional MRI marker may predict the outcome of electroconvulsive therapy in severe and treatment-resistant depression," Molecular Psychiatry, vol. 20, no. 5, pp. 609-614, 2015.

[47] A. Merkl, F. Schubert, A. Quante et al., "Abnormal cingulate and prefrontal cortical neurochemistry in major depression after 
electroconvulsive therapy," Biological Psychiatry, vol. 69, no. 8, pp. 772-779, 2011.

[48] M. Semkovska and D. M. McLoughlin, "Objective cognitive performance associated with electroconvulsive therapy for depression: a systematic review and meta-analysis," Biological Psychiatry, vol. 68, no. 6, pp. 568-577, 2010. 

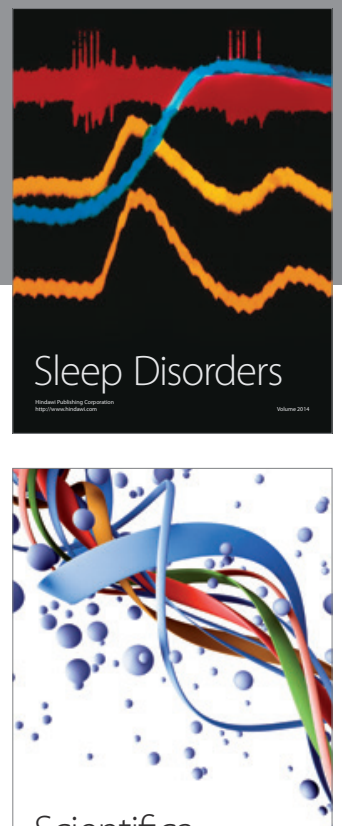

Scientifica
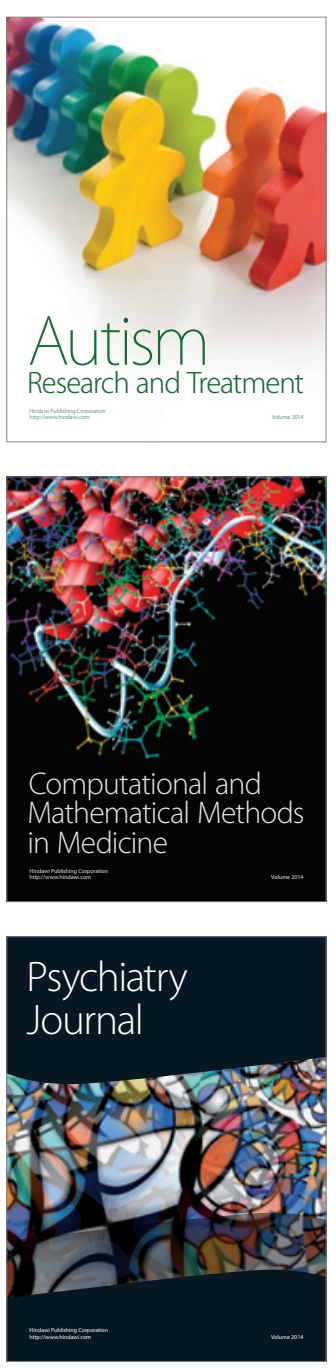
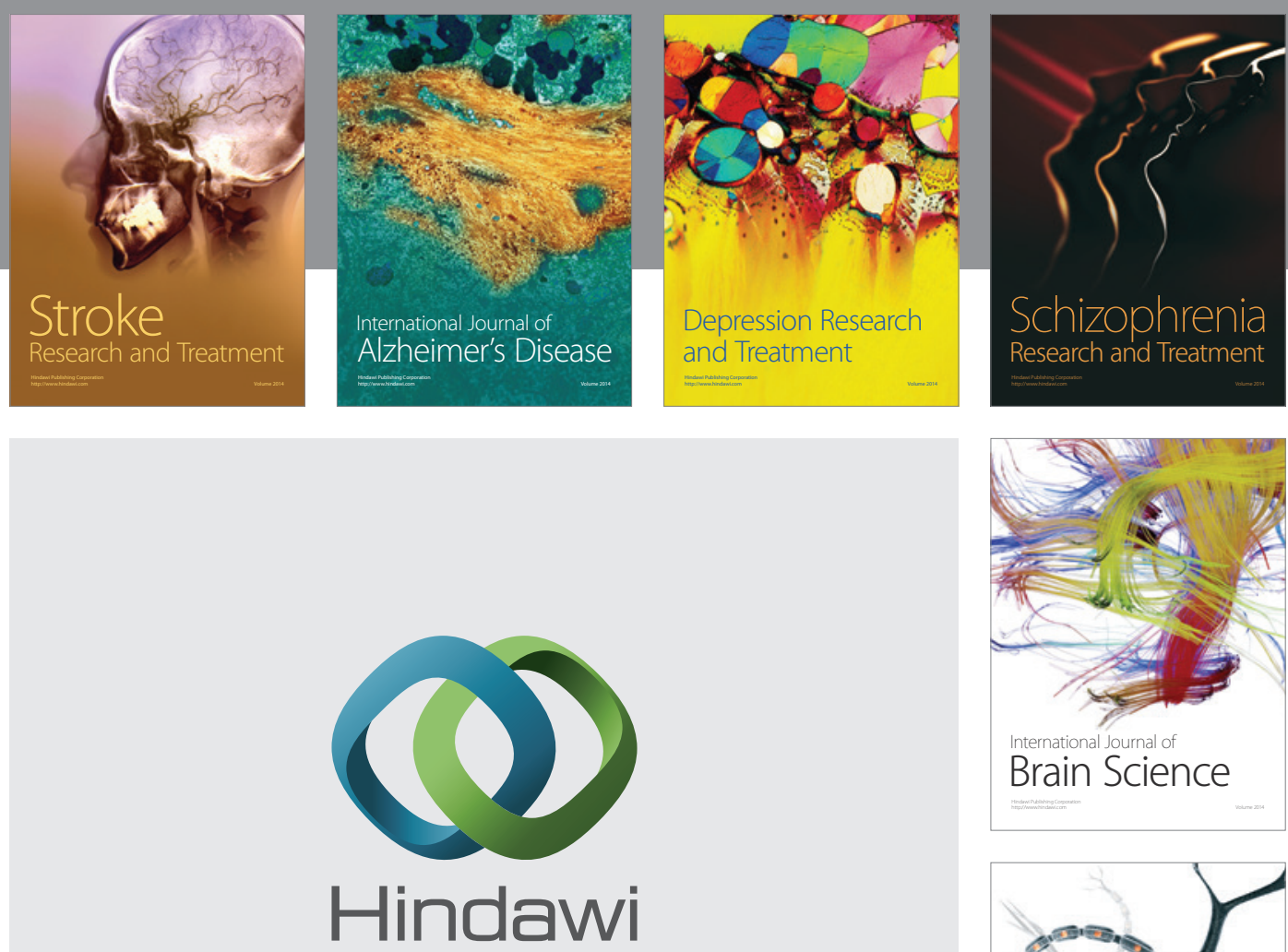

Submit your manuscripts at

http://www.hindawi.com
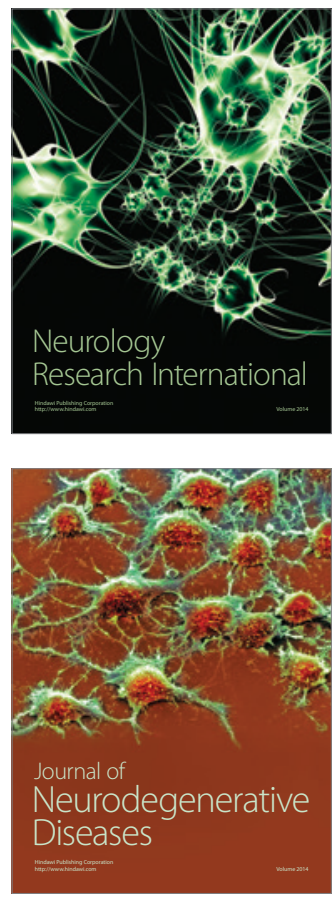

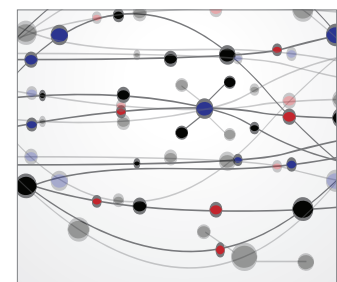

The Scientific World Journal
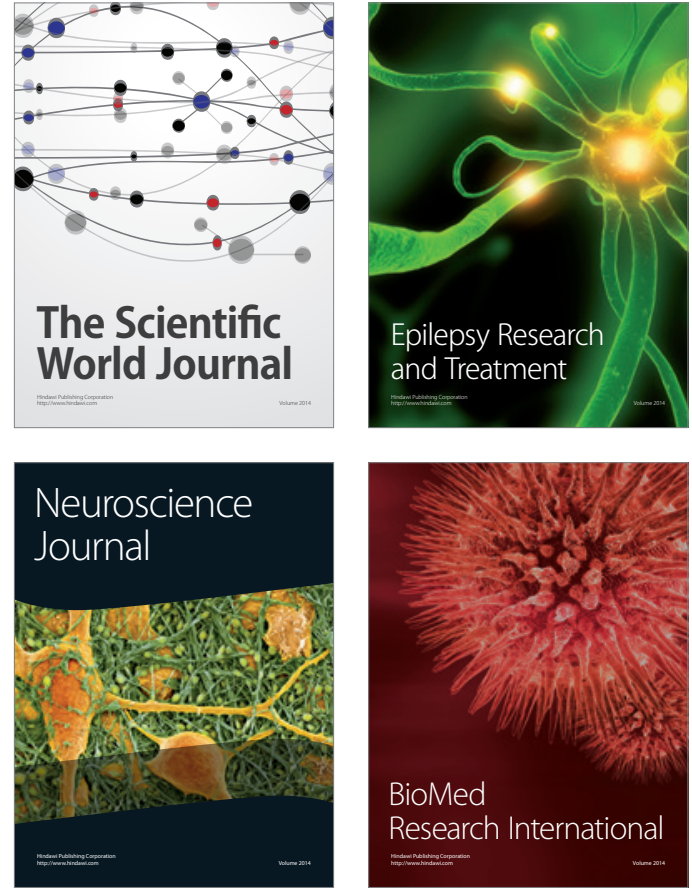

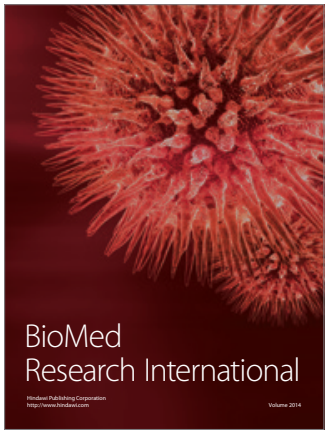

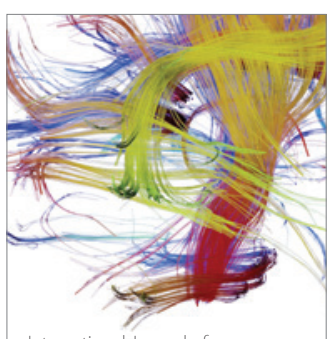

Brain Science

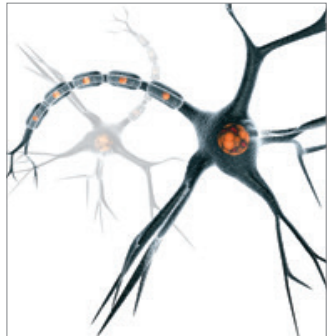

Neural Plasticity
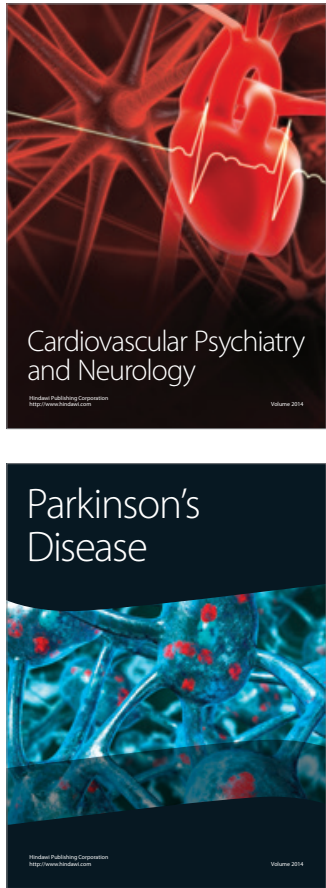